\title{
NON-EXACT INTEGRAL FUNCTORS
}

\author{
FERNANDO SANCHO DE SALAS
}

\begin{abstract}
We give a natural notion of (non-exact) integral functor $D_{\text {perf }}(X) \rightarrow$ $D_{c}^{b}(Y)$ in the context of $k$-linear and graded categories. In this broader sense, we prove that every $k$-linear and graded functor is integral.
\end{abstract}

\section{INTRODUCTION}

Let $k$ be a field, $X$ and $Y$ two projective $k$-schemes and $K$ an object of $D_{c}^{b}\left(X \times_{k} Y\right)$. Let us denote $p: X \times Y \rightarrow Y$ and $q: X \times Y \rightarrow X$ the natural projections. One has a functor

$$
\begin{aligned}
\Phi_{K}: D_{\text {perf }}(X) & \rightarrow D_{c}^{b}(Y), \\
M & \mapsto p_{*}\left(K \otimes q^{*} M\right)
\end{aligned}
$$

This functor is $k$-linear, graded and exact. We shall say that $\Phi_{K}$ is an exact integral functor of kernel $K$. We have then a functor

$$
\begin{aligned}
\Phi: D_{c}^{b}(X \times Y) & \rightarrow \operatorname{Hom}_{k}^{\text {ex }}\left(D_{\operatorname{perf}}(X), D_{c}^{b}(Y)\right) \\
K & \mapsto \Phi_{K}
\end{aligned}
$$

where $\operatorname{Hom}_{k}^{\text {ex }}\left(D_{\text {perf }}(X), D_{c}^{b}(Y)\right)$ denotes the category of $k$-linear, graded and exact functors (with $k$-linear and graded natural transformations). This functor is, in general, neither essentially injective or full (see [7]) or faithful (see [4]). However, one of the main open questions is whether it is essentially surjective. One has a positive answer for fully faithful functors $D_{\text {perf }}(X) \rightarrow D_{\text {perf }}(Y)$ due to Orlov and Lunts (see [9] and [11). More generally, A. Canonaco and P. Stellari have shown in [5] (see also [6] for the result in the supported case) that any exact functor $F: D^{b}(X) \rightarrow D^{b}(Y)$ satisfying

$$
\operatorname{Hom}_{D_{c}^{b}(Y)}(F(A), F(B)[k])=0
$$

for any sheaves $A$ and $B$ on $X$ and any integer $k<0$, is integral. There are also generalizations of the fully faithful case to derived stacks (see [10]) and twisted categories (see [5]).

The best evidence for a positive answer in general is due to the results of Töen concerning dg-categories. Indeed, it is proved in [13] that all dg (quasi-)functors between the dg-categories of perfect complexes on smooth proper schemes are of Fourier-Mukai type. This result, together with the conjecture by Bondal, Larsen and Lunts in [2] that states that all exact functors between the bounded derived categories of coherent sheaves on smooth projective varieties should be liftable to dg (quasi-)functors between the corresponding dg-enhancements, would give a positive answer to the question.

In this paper we show that if we work in the context of $k$-linear and graded categories and $k$-linear and graded functors (i.e., we forget exactness) the answer is positive. Of

Date: September 25, 2018.

2000 Mathematics Subject Classification. Primary 14F05; Secondary 18 E30.

Key words and phrases. derived categories, integral functors, linear functors.

Work supported by research projects MTM2009-07289 (MEC) and GR46 (JCYL). 
course we have to say what a $k$-linear and graded (may be non exact) integral functor means. The idea is very simple: since an object $K \in D_{c}^{b}(X \times Y)$ may be thought of as an exact functor $D_{\text {perf }}(X \times Y) \rightarrow D_{\text {perf }}(k)$, we shall instead consider, as a kernel, a $k$-linear and graded (may be non exact) functor $\omega: D_{\text {perf }}(X \times Y) \rightarrow D(k)$. Let us be more precise:

For any $k$-scheme $f: Z \rightarrow$ Spec $k$, let us denote $D_{\text {perf }}(Z)^{*}$ the category of $k$-linear and graded functors $D_{\text {perf }}(Z) \rightarrow D(k)$. One has a natural functor, $D_{c}^{b}(Z) \rightarrow D_{\text {perf }}(Z)^{*}$, $K \mapsto \omega_{K}$, where $\omega_{K}$ is the exact integral functor of kernel $K$, i.e., $\omega_{K}(M)=f_{*}(K \otimes M)$. This functor is fully faithful and its essential image is the subcategory $D_{\text {perf }}(Z)^{\vee}$ of exact and perfect functors (perfect means that it takes values in $D_{\text {perf }}(k)$ ).

Now, let $\omega \in D_{\text {perf }}(X \times Y)^{*}$. It induces, in the obvious way, a $k$-linear and graded functor

$$
\begin{aligned}
\Phi_{\omega}: D_{\text {perf }}(X) & \rightarrow D_{\text {perf }}(Y)^{*} \\
M & \mapsto \Phi_{\omega}(M)
\end{aligned}
$$

i.e., $\Phi_{\omega}(M)(N)=\omega\left(q^{*} M \otimes p^{*} N\right)$ (see also (1.1) for an alternative description which is closer to the usual definition). We shall say that $\Phi_{\omega}$ is an integral functor of kernel $\omega$. If $\omega$ is exact and perfect, then $\omega \simeq \omega_{K}$ for an unique $K \in D_{c}^{b}(X \times Y)$. Then $\Phi_{\omega}$ takes values in $D_{\text {perf }}(Y)^{\vee} \simeq D_{c}^{b}(Y)$ and $\Phi_{\omega} \simeq \Phi_{K}$.

One has then a functor

$$
\begin{aligned}
\Phi: D_{\operatorname{perf}}(X \times Y)^{*} & \rightarrow \operatorname{Hom}_{k}\left(D_{\operatorname{perf}}(X), D_{\operatorname{perf}}(Y)^{*}\right) \\
\omega & \mapsto \Phi_{\omega}
\end{aligned}
$$

extending the functor $\Phi: D_{c}^{b}(X \times Y) \rightarrow \mathbf{H o m}_{k}^{\mathrm{ex}}\left(D_{\mathrm{perf}}(X), D_{c}^{b}(Y)\right)$.

The aim of this paper is to prove that $\Phi: D_{\text {perf }}(X \times Y)^{*} \rightarrow \operatorname{Hom}_{k}\left(D_{\text {perf }}(X), D_{\text {perf }}(Y)^{*}\right)$ is essentially surjective. Even more, we shall construct a right inverse of $\Phi$, i.e., a functor

$$
\Psi: \operatorname{Hom}_{k}\left(D_{\text {perf }}(X), D_{\text {perf }}(Y)^{*}\right) \rightarrow D_{\text {perf }}(X \times Y)^{*}
$$

such that $\Phi \circ \Psi$ is isomorphic to the identity. In other words, for any $k$-linear and graded functor $F: D_{\text {perf }}(X) \rightarrow D_{\text {perf }}(Y)^{*}$ there exist a kernel $\omega_{F} \in D_{\text {perf }}(X \times Y)^{*}$ and an isomorphism $F \simeq \Phi_{\omega_{F}}$ which are functorial on $F$. This will be a consequence of an extension theorem (Theorem 2.8) that states that if $F: D_{\text {perf }}(X) \rightarrow D_{\text {perf }}(Y)^{*}$ is a $k$-linear and graded functor and $S$ is any $k$-scheme, then $F$ can be lifted to an $S$-linear functor $F_{S}: D_{\text {fhd } / X, S}(X \times S) \rightarrow D_{\text {perf }}(Y \times S)^{*}$, where $D_{\text {fhd } / X, S}(X \times S)$ is the category of objects in $D_{c}^{b}(X \times S)$ of finite homological dimension over both $X$ and $S$ (see Definition 2.6).

Let us denote $D_{\text {perf }}(X \times Y)^{Y-\vee}$ the full subcategory of $D_{\text {perf }}(X \times Y)^{*}$ whose objects are the $\omega \in D_{\text {perf }}(X \times Y)^{*}$ which are exact and perfect on $Y$, i.e. such that for any $M \in$ $D_{\text {perf }}(X), \Phi_{\omega}(M)$ belongs to $D_{\text {perf }}(Y)^{\vee}$. Taking into account the equivalence $D_{c}^{b}(Y) \stackrel{\sim}{\rightarrow}$ $D_{\text {perf }}(Y)^{\vee}$, we obtain functors $\Phi: D_{\text {perf }}(X \times Y)^{Y-\vee} \rightarrow \mathbf{H o m}_{k}\left(D_{\text {perf }}(X), D_{c}^{b}(Y)\right)$ and $\Psi: \operatorname{Hom}_{k}\left(D_{\text {perf }}(X), D_{c}^{b}(Y)\right) \rightarrow D_{\text {perf }}(X \times Y)^{Y-\vee}$, such that $\Phi \circ \Psi$ is isomorphic to the identity. Finally, if we denote $D_{\text {perf }}(X \times Y)^{\text {bi- }}\left(\right.$ the full subcategory of $D_{\text {perf }}(X \times$ $Y)^{*}$ whose objects are the $\omega \in D_{\text {perf }}(X \times Y)^{*}$ which are bi-exact (see Definition 2.17), then we obtain functors $\Phi: D_{\text {perf }}(X \times Y)^{\mathrm{bi}-\vee} \rightarrow \mathbf{H o m}_{k}^{\mathrm{ex}}\left(D_{\text {perf }}(X), D_{c}^{b}(Y)\right)$ and $\Psi: \operatorname{Hom}_{k}^{\text {ex }}\left(D_{\text {perf }}(X), D_{c}^{b}(Y)\right) \rightarrow D_{\text {perf }}(X \times Y)^{\mathrm{bi}-\vee}$, such that $\Phi \circ \Psi$ is isomorphic to the identity.

We shall also give these results in the relative setting. That is, assume that $X$ and $Y$ are flat $T$-schemes and let $p: X \times_{T} Y \rightarrow Y, q: X \times_{T} Y \rightarrow X$ be the natural projections. 
For each $M \in D_{c}^{b}\left(X \times_{T} Y\right)$ one has a $T$-linear functor $\Phi_{K}: D_{\text {perf }}(X) \rightarrow D_{c}^{b}(Y), M \mapsto$ $p_{*}\left(K \otimes q^{*} M\right)$; one has then a $T$-linear functor

$$
\Phi: D_{c}^{b}\left(X \times_{T} Y\right) \rightarrow \mathbf{H o m}_{T}^{\mathrm{ex}}\left(D_{\mathrm{perf}}(X), D_{c}^{b}(Y)\right)
$$

More generally, for any $\omega \in D_{\text {perf }}\left(X \times_{T} Y\right)^{*}$ one has a $T$-linear functor $\Phi_{\omega}: D_{\text {perf }}(X) \rightarrow$ $D_{\text {perf }}(Y)^{*}$; one has then a $T$-linear functor

$$
\Phi: D_{\text {perf }}\left(X \times_{T} Y\right)^{*} \rightarrow \operatorname{Hom}_{T}\left(D_{\text {perf }}(X), D_{\text {perf }}(Y)^{*}\right) .
$$

As before, we shall construct a $T$-linear functor

$$
\Psi: \operatorname{Hom}_{T}\left(D_{\text {perf }}(X), D_{\text {perf }}(Y)^{*}\right) \rightarrow D_{\text {perf }}\left(X \times_{T} Y\right)^{*}
$$

such that $\Phi \circ \Psi$ is isomorphic to the identity.

\section{Notations AND BASiC RESUlts}

Throughout the paper $k$ denotes a field. All the schemes are assumed to be proper $k$-schemes. If $f: X \rightarrow Y$ is a morphism of $T$-schemes, we shall still denote by $f$ the morphism $X \times_{T} T^{\prime} \rightarrow Y \times_{T} T^{\prime}$ induced by $f$ after a base change $T^{\prime} \rightarrow T$.

For a scheme $X$, we denote by $D(X)$ the derived category of complexes of $\mathcal{O}_{X^{-}}$ modules with quasi-coherent cohomology, $D_{c}^{b}(X)$ the full subcategory of complexes with bounded and coherent cohomology and $D_{\text {perf }}(X)$ the full subcategory of perfect complexes.

Since we shall deal with derived categories, we shall use the abbreviated notations $f_{*}, f^{*}, \otimes, \ldots$ for the derived functors $\mathbf{R} f_{*}, \mathbf{L} f^{*}, \stackrel{\mathbf{L}}{\otimes}, \ldots$

We shall use extensively the following results of derived categories:

(1) Projection formula: If $f: X \rightarrow Y$ is a morphism of schemes, then one has a natural isomorphism $f_{*}\left(M \otimes f^{*} L\right) \simeq\left(f_{*} M\right) \otimes L$, with $M \in D(X), L \in D(Y)$.

(2) Flat base change: Let us consider a cartesian diagram

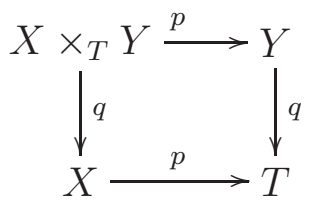

with $p$ flat. For any $N \in D(Y)$ one has a natural isomorphism $p^{*} q_{*} N \stackrel{\sim}{\rightarrow} q_{*} p^{*} N$.

\subsection{Non-exact integral functors.}

Let $k$ be a field, $D(k)$ the derived category of complexes of $k$-vector spaces.

Definition 1.1. Let $p: Z \rightarrow$ Spec $k$ be a $k$-scheme. A linear form on $Z$ is a $k$-linear and graded functor $\omega: D_{\text {perf }}(Z) \rightarrow D(k)$. We say that $\omega$ is perfect if it takes values in $D_{\text {perf }}(k)$. We say that $\omega$ is exact if it takes exact triangles into exact triangles.

A linear morphism $\omega \rightarrow \omega^{\prime}$ between linear forms on $Z$ is just a morphism of $k$-linear and graded functors. We shall denote by $D_{\text {perf }}(Z)^{*}$ the category of $k$-linear forms on $Z$ and $k$-linear morphisms and by $D_{\text {perf }}(Z)^{\vee}$ the full subcategory of $D_{\text {perf }}(Z)^{*}$ whose objects are the exact and perfect linear forms on $Z$. Both $D_{\text {perf }}(Z)^{*}$ and $D_{\text {perf }}(Z)^{\vee}$ are $k$-linear and graded categories.

For any $K \in D_{c}^{b}(Z)$ one has a (perfect and exact) linear form $\omega_{K}$ on $Z$, defined by $\omega_{K}(M)=p_{*}(K \otimes M)$. Moreover one has the following 
Proposition 1.2. Let $Z$ be a projective $k$-scheme. The functor $D_{c}^{b}(Z) \rightarrow D_{\text {perf }}(Z)^{\vee}$, $K \mapsto \omega_{K}$, is an equivalence (of $k$-linear and graded categories).

Proof. It is proved in 3 , that any contravariant cohomological functor of finite type over $D_{\text {perf }}(Z)$ ( $Z$ a projective scheme over $k$ ) is representable by a bounded complex with coherent homology. It follows that if $\omega: D_{\text {perf }}(Z) \rightarrow D_{\text {perf }}(k)$ is exact, then it has a right pseudo adjoint $\omega^{\#}: D_{\text {perf }}(k) \rightarrow D_{c}^{b}(Z)$; that is, one has

$$
\operatorname{Hom}_{D(k)}(\omega(M), E) \simeq \operatorname{Hom}_{D(Z)}\left(M, \omega^{\#}(E)\right)
$$

for any $M \in D_{\text {perf }}(Z), E \in D_{\text {perf }}(k)$. Since $\omega^{\#}$ is $k$-linear and graded, one has $\omega^{\#}(E) \simeq$ $\omega^{\#}(k) \otimes p^{*} E$, and then $\omega \simeq \omega_{K}$ with $K=\mathbf{R} \mathcal{H} o m\left(\omega^{\#}(k), p^{!} k\right)$. Conclusion follows (see [12] for further details and a more general statement).

Definition 1.3. Tensor product, direct and inverse image.

(1) $D_{\text {perf }}(Z)^{*}$ has a $D_{\text {perf }}(Z)$-module structure: for any $M \in D_{\text {perf }}(Z), \omega \in D_{\text {perf }}(Z)^{*}$, we define $\omega \otimes M \in D_{\text {perf }}(Z)^{*}$ by the formula $(\omega \otimes M)(N)=\omega(M \otimes N)$.

(2) For any morphism of $k$-schemes $f: Z \rightarrow Z^{\prime}$, we define $f_{*}: D_{\text {perf }}(Z)^{*} \rightarrow D_{\text {perf }}\left(Z^{\prime}\right)^{*}$ as the $k$-linear and graded functor induced by $f^{*}: D_{\text {perf }}\left(Z^{\prime}\right) \rightarrow D_{\text {perf }}(Z)$; that is, $\left(f_{*} \omega\right)\left(M^{\prime}\right)=\omega\left(f^{*} M^{\prime}\right)$, for $M^{\prime} \in D_{\text {perf }}\left(Z^{\prime}\right), \omega \in D_{\text {perf }}(Z)^{*}$.

(3) If $f: Z \rightarrow Z^{\prime}$ is flat, we define $f^{*}: D_{\text {perf }}\left(Z^{\prime}\right)^{*} \rightarrow D_{\text {perf }}(Z)^{*}$ as the $k$-linear and graded functor induced by $f_{*}: D_{\text {perf }}(Z) \rightarrow D_{\text {perf }}\left(Z^{\prime}\right)$; that is, $\left(f^{*} \omega\right)(M)=$ $\omega\left(f_{*} M\right)$, for $M \in D_{\text {perf }}(Z), \omega \in D_{\text {perf }}\left(Z^{\prime}\right)^{*}$.

Definition 1.4. Let $p: X \rightarrow$ Spec $k$ and $q: Y \rightarrow$ Spec $k$ be two $k$-schemes and $p: X \times$ $Y \rightarrow Y, q: X \times Y \rightarrow X$ the natural projections. For each $\omega \in D_{\text {perf }}(X \times Y)^{*}$ we have a $k$-linear and graded functor

$$
\begin{aligned}
\Phi_{\omega}: D_{\text {perf }}(X) & \rightarrow D_{\text {perf }}(Y)^{*} \\
M & \mapsto \Phi_{\omega}(M)=p_{*}\left(\omega \otimes q^{*} M\right)
\end{aligned}
$$

We say that $\Phi_{\omega}$ is an integral functor of kernel $\omega$.

Example 1.5. Assume that $X$ and $Y$ are projective $k$-schemes. If $\omega$ is exact and perfect, then $\omega \simeq \omega_{K}$ for a unique $K \in D_{c}^{b}(X \times Y)$ by Proposition 1.2, Then $\Phi_{\omega}$ takes values in $D_{\text {perf }}(Y)^{\vee} \simeq D_{c}^{b}(Y)$ and $\Phi_{\omega}$ is isomorphic to the usual exact integral functor $\Phi_{K}$. $\triangle$

Let us denote $\operatorname{Hom}_{k}\left(D_{\text {perf }}(X), D_{\text {perf }}(Y)^{*}\right)$ the category of $k$-linear and graded functors from $D_{\text {perf }}(X)$ to $D_{\text {perf }}(Y)^{*}$ and $k$-linear and graded morphisms of functors. It is a $k$-linear and graded category in the obvious way. One has a $k$-linear and graded functor

$$
\begin{aligned}
\Phi: D_{\text {perf }}(X \times Y)^{*} & \rightarrow \operatorname{Hom}_{k}\left(D_{\text {perf }}(X), D_{\text {perf }}(Y)^{*}\right) \\
\omega & \mapsto \Phi_{\omega}
\end{aligned}
$$

and a commutative diagram

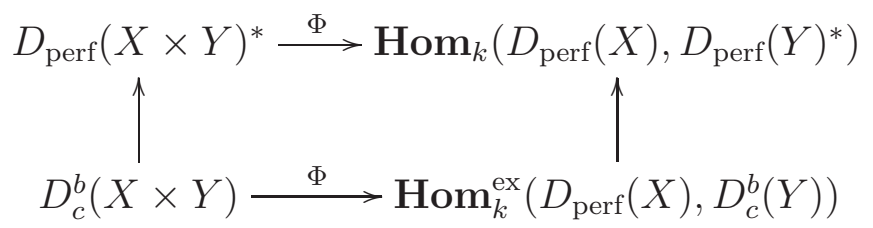

whose vertical maps are fully faithful. 


\section{MAin Results}

The aim of this section is to construct a functor $\Psi: \operatorname{Hom}_{k}\left(D_{\text {perf }}(X), D_{\text {perf }}(Y)^{*}\right) \rightarrow$ $D_{\text {perf }}(X \times Y)^{*}$ such that $\Phi \circ \Psi$ is isomorphic to the identity. This will be a consequence of the following extension theorem: a $k$-linear and graded functor $F: D_{\text {perf }}(X) \rightarrow$ $D_{\text {perf }}(Y)^{*}$ can be (functorially) extended to an $S$-linear functor $F_{S}: D_{\text {fhd } / X, S}(X \times S) \rightarrow$ $D_{\text {perf }}(Y \times S)^{*}$, for any $k$-scheme $S$ (see Definition 2.6 for the meaning of $D_{\text {fhd } / X, S}(X \times$ $S))$.

\subsection{Cokernels of linear forms.}

Let $E_{\bullet}=\left\{E_{1} \underset{\mathrm{d}_{1}}{\stackrel{\mathrm{d}_{0}}{\longrightarrow}} E_{0}\right\}$ be two maps in $D(k)$. We define Coker $\left(E_{\bullet}\right)$ as the cokernel of the morphisms of vector spaces $H\left(E_{1}\right) \underset{H\left(\mathrm{~d}_{1}\right)}{\stackrel{H\left(\mathrm{~d}_{0}\right)}{\longrightarrow}} H\left(E_{0}\right)$.

Now let $p: Z \rightarrow$ Spec $k$ be a $k$-scheme and let $\omega_{\bullet}=\left\{\omega_{1} \stackrel{\stackrel{\mathrm{d}_{0}}{\longrightarrow}}{\rightarrow} \omega_{0}\right\}$ be two morphisms in $D_{\text {perf }}(Z)^{*}$. For each $M \in D_{\text {perf }}(Z)$, let us denote $\omega_{\bullet}(M)=\left\{\omega_{1}(M) \underset{\mathrm{d}_{1}(M)}{\stackrel{\mathrm{d}_{0}(M)}{\longrightarrow}} \omega_{0}(M)\right\}$. We define $\operatorname{Coker}\left(\omega_{\bullet}\right)$ as the object in $D_{\text {perf }}(Z)^{*}$ defined by

$$
\operatorname{Coker}\left(\omega_{\bullet}\right)(M)=\operatorname{Coker}\left(\omega_{\bullet}(M)\right)
$$

This is clearly functorial on $\omega_{\bullet}$. The next proposition is immediate.

Proposition 2.1. Let $\omega_{\bullet}=\left\{\omega_{1} \underset{\mathrm{d}_{1}}{\stackrel{\mathrm{d}_{0}}{\longrightarrow}} \omega_{0}\right\}$ be two morphisms in $D_{\text {perf }}(Z)^{*}$.

(1) For any $M \in D_{\text {perf }}(Z)$ one has $\operatorname{Coker}\left(\omega_{\bullet} \otimes M\right)=\operatorname{Coker}\left(\omega_{\bullet}\right) \otimes M$.

(2) For any morphism $f: Z \rightarrow Z^{\prime}$, one has $f_{*} \operatorname{Coker}\left(\omega_{\bullet}\right)=\operatorname{Coker}\left(f_{*} \omega_{\bullet}\right)$.

\subsection{The perfect-resolution.}

Let $p: X \rightarrow$ Spec $k, f: S \rightarrow$ Spec $k$ be two $k$-schemes and $M \in D_{c}^{b}(X \times S)$ an object of finite homological dimension over $S$ (see Definition 2.6). We still denote by $p: X \times S \rightarrow S$ and $f: X \times S \rightarrow X$ the natural projections. For each $\mathcal{E} \in D_{\text {perf }}(X)$ we shall denote $R_{\mathcal{E}}(M)=f^{*} \mathcal{E} \otimes p^{*} p_{*}\left(f^{*} \mathcal{E}^{*} \otimes M\right)$, with $\mathcal{E}^{*}=\mathbf{R} \mathcal{H} o m_{X}^{\bullet}\left(\mathcal{E}, \mathcal{O}_{X}\right)$. One has a natural morphism $\rho_{M}^{\mathcal{E}}: R_{\mathcal{E}}(M) \rightarrow M$. We shall denote

$$
R_{0}(M)=\underset{\mathcal{E} \in D_{\text {perf }}(X)}{\oplus} R_{\mathcal{E}}(M)
$$

and $\rho_{M}: R_{0}(M) \rightarrow M$ the natural map. This is functorial on $M$.

Let $R_{1}(M)=R_{0}\left(R_{0}(M)\right)$. One has two morphisms

$$
R_{1}(M) \underset{\mathrm{d}_{1}}{\stackrel{\mathrm{d}_{0}}{\longrightarrow}} R_{0}(M)
$$

namely: $\mathrm{d}_{0}=\rho_{R_{0}(M)}$ and $\mathrm{d}_{1}=R_{0}\left(\rho_{M}\right)$. It is immediate to check that $\rho_{M} \circ \mathrm{d}_{0}=\rho_{M} \circ \mathrm{d}_{1}$.

More explicitly, $R_{1}(M)=\underset{\mathcal{E}_{1}, \mathcal{E}_{0} \in D_{\text {perf }}(X)}{\oplus} R_{\mathcal{E}_{1}} R_{\mathcal{E}_{0}}(M)$ and

$$
R_{\mathcal{E}_{1}} R_{\mathcal{E}_{0}}(M) \simeq f^{*} \mathcal{E}_{1} \otimes p^{*} p_{*} f^{*}\left(\mathcal{E}_{1}^{*} \otimes \mathcal{E}_{0}\right) \otimes p^{*} p_{*}\left(f^{*} \mathcal{E}_{0}^{*} \otimes M\right) .
$$


The differentials $\mathrm{d}_{0}, \mathrm{~d}_{1}: R_{1}(M) \rightarrow R_{0}(M)$ are induced by the morphisms

$$
\begin{gathered}
\rho_{R_{\mathcal{E}_{0}}(M)}^{\mathcal{E}_{1}}: R_{\mathcal{E}_{1}} R_{\mathcal{E}_{0}}(M) \rightarrow R_{\mathcal{E}_{0}}(M) \\
R_{\mathcal{E}_{1}}\left(\rho_{M}^{\mathcal{E}_{0}}\right): R_{\mathcal{E}_{1}} R_{\mathcal{E}_{0}}(M) \rightarrow R_{\mathcal{E}_{1}}(M)
\end{gathered} .
$$

Proposition 2.2. For any $L \in D_{\text {perf }}(X), R_{1}\left(f^{*} L\right) \underset{\mathrm{d}_{1}}{\stackrel{\mathrm{d}_{0}}{\longrightarrow}} R_{0}\left(f^{*} L\right) \stackrel{\rho_{f^{*} L}}{\longrightarrow} f^{*} L$ is exact.

Proof. The morphism $\rho_{f^{*} L}^{f^{*} L}: R_{f^{*} L}\left(f^{*} L\right) \rightarrow f^{*} L$ has a natural section $h: f^{*} L \rightarrow R_{f^{*} L}\left(f^{*} L\right)=$ $f^{*} L \otimes p^{*} p_{*}\left(f^{*} L^{*} \otimes f^{*} L\right)$ induced by the natural map $\mathcal{O}_{S} \rightarrow p_{*}\left(f^{*} L^{*} \otimes f^{*} L\right)$. Then we have a map $h_{0}: f^{*} L \rightarrow R_{0}\left(f^{*} L\right)$ which is $h$ in the $f^{*} L$-component and zero in the others. It is clear that $h_{0}$ is a section of $\rho_{f^{*} L}$. Now define $h_{1}: R_{0}\left(f^{*} L\right) \rightarrow R_{1}\left(f^{*} L\right)$ as $h_{1}=-R_{0}(h)$. One can check that $\left(\mathrm{d}_{0}-\mathrm{d}_{1}\right) \circ h_{1}+h_{0} \circ \rho_{f^{*} L}=\mathrm{Id}$, hence the result.

We shall denote $R_{\bullet}^{X \times S / S}(M):=\left\{R_{1}(M) \underset{\mathrm{d}_{1}}{\stackrel{\mathrm{d}_{0}}{\longrightarrow}} R_{0}(M)\right\}$.

Remark 2.3. If $G: D_{\text {perf }}(X \times S) \rightarrow \mathcal{D}$ is an additive functor and $\mathcal{D}$ has infinite direct sums, we can define $G\left(R_{1}(M)\right):=\underset{\mathcal{E}_{1} \mathcal{E}_{0}}{\oplus} G\left(R_{\mathcal{E}_{1}} R_{\mathcal{E}_{0}}(M)\right)$ and $G\left(R_{0}(M)\right):=\underset{\mathcal{E}}{\oplus} G\left(R_{\mathcal{E}}(M)\right)$. One has differentials $G\left(\mathrm{~d}_{0}\right), G\left(\mathrm{~d}_{1}\right): G\left(R_{1}(M)\right) \rightarrow G\left(R_{0}(M)\right)$. We shall denote

$$
G\left(R_{\bullet}(M)\right)=\left\{G\left(R_{1}(M)\right) \underset{G\left(\mathrm{~d}_{1}\right)}{\stackrel{G\left(\mathrm{~d}_{0}\right)}{\longrightarrow}} G\left(R_{0}(M)\right)\right\} .
$$

For any $L \in D_{\text {perf }}(X), G\left(R_{1}\left(f^{*} L\right)\right) \underset{G\left(\mathrm{~d}_{1}\right)}{\stackrel{G\left(\mathrm{~d}_{0}\right)}{\longrightarrow}} G\left(R_{0}\left(f^{*} L\right)\right) \stackrel{G\left(\rho_{f^{*} L}\right)}{\longrightarrow} G\left(f^{*} L\right)$ is exact.

In the next propositions we shall prove the $S$-linearity of $R_{\bullet}^{X \times S / S}(M)$ and its compatibility with direct images.

Proposition 2.4. For any $V \in D_{\text {perf }}(S)$ one has a natural isomorphism $R_{\bullet}^{X \times S / S}(M \otimes$ $\left.p^{*} V\right) \stackrel{\sim}{\rightarrow} R_{\bullet}^{X \times S / S}(M) \otimes p^{*} V$.

Proof. One has a natural isomorphism $R_{\mathcal{E}}\left(M \otimes p^{*} V\right) \simeq R_{\mathcal{E}}(M) \otimes p^{*} V$. Indeed, by projection formula,

$$
\begin{aligned}
R_{\mathcal{E}}\left(M \otimes p^{*} V\right)=f^{*} \mathcal{E} \otimes p^{*} p_{*}\left(f^{*} \mathcal{E}^{*} \otimes M \otimes p^{*} V\right) & \simeq f^{*} \mathcal{E} \otimes p^{*} p_{*}\left(f^{*} \mathcal{E}^{*} \otimes M\right) \otimes p^{*} V \\
& =R_{\mathcal{E}}(M) \otimes p^{*} V .
\end{aligned}
$$

One checks that the diagram

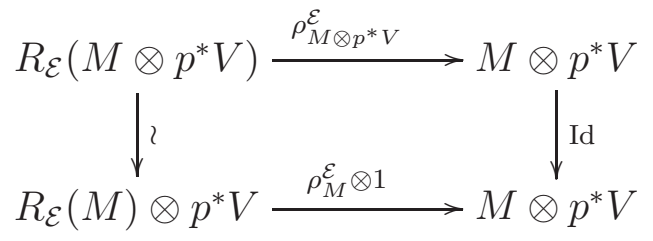

is commutative. Hence one has an isomorphism $R_{0}\left(M \otimes p^{*} V\right) \stackrel{\sim}{\rightarrow} R_{0}(M) \otimes p^{*} V$ and a commutative diagram 


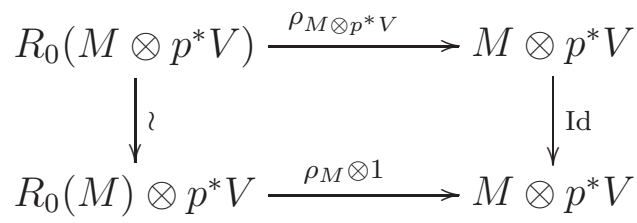

Conclusion follows.

Proposition 2.5. One has a natural isomorphism $f_{*}\left[R_{\bullet}^{X \times S / S}(M)\right] \simeq R_{\bullet}^{X / k}\left(f_{*} M\right)$.

Proof. By projection formula and flat base change one has

$$
\begin{aligned}
f_{*} R_{\mathcal{E}}(M)=f_{*}\left[f^{*} \mathcal{E} \otimes p^{*} p_{*}\left(f^{*} \mathcal{E}^{*} \otimes M\right)\right] \simeq \mathcal{E} \otimes f_{*} p^{*} p_{*}\left(f^{*} \mathcal{E}^{*} \otimes M\right) & \simeq \mathcal{E} \otimes p^{*} p_{*}\left(\mathcal{E}^{*} \otimes f_{*} M\right) \\
& =R_{\mathcal{E}}\left(f_{*} M\right)
\end{aligned}
$$

Moreover, the diagram

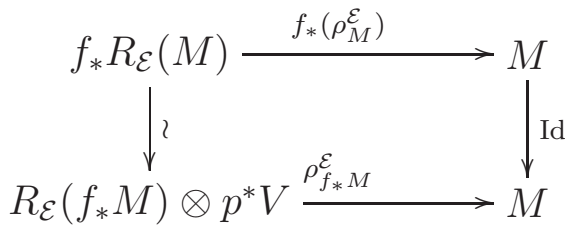

is commutative. One has then $f_{*} R_{0}(M) \simeq R_{0}\left(f_{*} M\right)$ and a commutative diagram

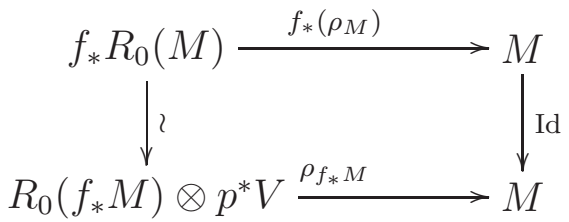

Conclusion follows.

\subsection{The extension theorem.}

We need to introduce a relative notion of perfectness.

Definition 2.6. Let $f: Z \rightarrow T$ be a morphism of schemes. An object $M \in D_{c}^{b}(Z)$ is said to be of finite homological dimension over $T$ (fhd $/ T$ for short), if $M \otimes f^{*} N$ is bounded and coherent for any $N \in D_{c}^{b}(T)$. We shall denote by $D_{\text {fhd } / T}(Z)$ the faithful subcategory of the objects of finite homological dimension over $T$.

The following properties of fhd-objects are quite immediate (see [ㅇ, Section 1.2]).

Proposition 2.7. (1) If $f$ is flat, then $D_{\text {perf }}(Z) \subset D_{\text {fhd } / T}(Z)$.

(2) If $M$ is fhd over $T$ and $f$ is proper, then $f_{*} M$ is perfect.

(3) If $M$ is fhd over $T$ and $\mathcal{E} \in D_{\text {perf }}(Z)$, then $M \otimes \mathcal{E}$ is fhd over $T$.

Given two schemes $X$ and $S$, we shall denote by $D_{\text {fhd } / X, S}(X \times S)$ the category of objects in $D_{c}^{b}(X \times S)$ of finite homological dimension over both $X$ and $S$.

Theorem 2.8. Let $p: X \rightarrow \operatorname{Spec} k$ and $q: Y \rightarrow$ Spec $k$ be two proper $k$-schemes. Let $F: D_{\text {perf }}(X) \rightarrow D_{\text {perf }}(Y)^{*}$ be a $k$-linear and graded functor. For any proper $k$-scheme $f: S \rightarrow$ Spec $k$ there exists a functor

$$
F_{S}: D_{\text {fhd } / X, S}(X \times S) \rightarrow D_{\text {perf }}(Y \times S)^{*}
$$

such that: 
1) $F_{S}$ is $S$-linear: one has a bi-functorial isomorphism $F_{S}\left(M \otimes p^{*} V\right) \simeq F_{S}(M) \otimes q^{*} V$ for any $M \in D_{\text {fhd } / X, S}(X \times S), V \in D_{\text {perf }}(S)$.

2) It is compatible with $F$ : for any $M \in D_{\text {fhd } / X, S}(X \times S)$ one has a natural isomorphism $f_{*} F_{S}(M) \simeq F\left(f_{*} M\right)$.

3) $F_{S}$ is functorial on $F$.

Proof. Let $M \in D_{\text {fhd } / X, S}(X \times S)$. Let us take the "perfect" resolution of $M, R_{\bullet}^{X \times S / S}(M)$, constructed in section 2.2, Recall that $R_{\mathcal{E}}(M)=f^{*} \mathcal{E} \otimes p^{*} p_{*}\left(f^{*} \mathcal{E}^{*} \otimes M\right)$. Since $M$ is fhd over $S, R_{\mathcal{E}}(M)$ belongs to $p^{*} D_{\text {perf }}(S) \otimes f^{*} D_{\text {perf }}(X)$. In particular, $R_{\mathcal{E}}(M)$ is fhd over $S$ and then $R_{\mathcal{E}_{1}} R_{\mathcal{E}_{0}}(M)$ belongs also to $p^{*} D_{\text {perf }}(S) \otimes f^{*} D_{\text {perf }}(X)$.

Taking in mind the explicit expression of $R_{\mathcal{E}}(M)$ and $R_{\mathcal{E}_{1}} R_{\mathcal{E}_{0}}(M)$ (see (2.1)), let us put

and

$$
\begin{aligned}
\tilde{F}\left(R_{\mathcal{E}}(M)\right) & :=f^{*} F(\mathcal{E}) \otimes q^{*} p_{*}\left(f^{*} \mathcal{E}^{*} \otimes M\right) \\
\tilde{F}\left(R_{\mathcal{E}_{1}} R_{\mathcal{E}_{0}}(M)\right) & :=f^{*} F\left(\mathcal{E}_{1}\right) \otimes q^{*} p_{*} f^{*}\left(\mathcal{E}_{1}^{*} \otimes \mathcal{E}_{0}\right) \otimes q^{*} p_{*}\left(f^{*} \mathcal{E}_{0}^{*} \otimes M\right)
\end{aligned}
$$

$$
\begin{aligned}
\tilde{F}\left(R_{0}(M)\right) & :=\underset{\mathcal{E} \in D_{\text {perf }}(X)}{\oplus} \tilde{F}\left(R_{\mathcal{E}}(M)\right) \\
\tilde{F}\left(R_{1}(M)\right) & :={ }_{\mathcal{E}_{1}, \mathcal{E}_{0} \in D_{\text {perf }}(X)}^{\oplus} \tilde{F}\left(R_{\mathcal{E}_{1}} R_{\mathcal{E}_{0}}(M)\right)
\end{aligned}
$$

By Lemma 2.9 (see below) the morphisms $\mathrm{d}_{0}, \mathrm{~d}_{1}: R_{1}(M) \rightarrow R_{0}(M)$ induce morphisms

$$
\tilde{\mathrm{d}}_{0}, \tilde{\mathrm{d}}_{1}: \tilde{F}\left(R_{1}(M)\right) \rightarrow \tilde{F}\left(R_{0}(M)\right)
$$

in $D_{\text {perf }}(Y \times S)^{*}$ which are functorial on $M$. Finally, we define

$$
F_{S}(M):=\operatorname{Coker}\left(\tilde{F}\left(R_{\bullet}(M)\right)\right.
$$

It is clear that $F_{S}(M)$ is functorial on $M$, hence we obtain a functor $F_{S}: D_{\text {perf }}(X \times S) \rightarrow$ $D_{\text {perf }}(Y \times S)^{*}$. By construction, $F_{S}$ satisfies 3$)$.

Lemma 2.9. Let $V, V^{\prime} \in D_{\text {perf }}(S)$ and $\mathcal{E}, \mathcal{E}^{\prime} \in D_{\text {perf }}(X)$. One has a natural map $\operatorname{Hom}_{D_{\text {perf }}(X \times S)}\left(p^{*} V \otimes f^{*} \mathcal{E}, p^{*} V^{\prime} \otimes f^{*} \mathcal{E}^{\prime}\right) \rightarrow \operatorname{Hom}_{D_{\text {perf }}(Y \times S)^{*}}\left(q^{*} V \otimes f^{*} F(\mathcal{E}), q^{*} V^{\prime} \otimes f^{*} F\left(\mathcal{E}^{\prime}\right)\right)$

This map is compatible with composition; moreover, it is $S$-linear and extends $F$ (the precise meaning of these will be given in the proof).

Proof. A morphism $h: p^{*} V \otimes f^{*} \mathcal{E} \rightarrow p^{*} V^{\prime} \otimes f^{*} \mathcal{E}^{\prime}$ corresponds with a morphism $\bar{h}: \mathcal{E} \rightarrow$ $\mathcal{E}^{\prime} \otimes_{k} \mathbf{R H o m}^{\bullet}\left(V, V^{\prime}\right)$. Since $F$ is $k$-linear and graded, it induces a morphism $F(\bar{h}): F(\mathcal{E}) \rightarrow$ $F\left(\mathcal{E}^{\prime}\right) \otimes_{k} \mathbf{R H o m}^{\bullet}\left(V, V^{\prime}\right)$. Hence, for any $N \in D_{\text {perf }}(Y \times S)$ one has morphisms

$$
\begin{aligned}
\left(q^{*} V\right. & \left.\otimes f^{*} F(\mathcal{E})\right)(N)=F(\mathcal{E})\left(f_{*}\left(q^{*} V \otimes N\right)\right) \stackrel{F(\bar{h})}{\rightarrow}\left[F\left(\mathcal{E}^{\prime}\right) \otimes_{k} \mathbf{R H o m} \cdot\left(V, V^{\prime}\right)\right]\left(f_{*}\left(q^{*} V \otimes N\right)\right) \\
& =F\left(\mathcal{E}^{\prime}\right)\left(f_{*}\left(q^{*} V \otimes N\right) \otimes_{k} \mathbf{R H o m} \cdot\left(V, V^{\prime}\right)\right) \stackrel{(*)}{\rightarrow} F\left(\mathcal{E}^{\prime}\right)\left(f_{*}\left(q^{*} V \otimes N\right)\right) \\
& =\left(q^{*} V^{\prime} \otimes f^{*} F\left(\mathcal{E}^{\prime}\right)\right)(N)
\end{aligned}
$$

where $\left(^{*}\right)$ is the morphism induced by the natural evaluation map $f_{*}\left(q^{*} V \otimes N\right) \otimes_{k}$ $\mathbf{R H o m}^{\bullet}\left(V, V^{\prime}\right) \rightarrow f_{*}\left(q^{*} V^{\prime} \otimes N\right)$. That is, one obtains a morphism $\tilde{h}: q^{*} V \otimes f^{*} F(\mathcal{E}) \rightarrow$ $q^{*} V^{\prime} \otimes f^{*} F\left(\mathcal{E}^{\prime}\right)$.

One can check from the construction that $\widetilde{(f \circ g)}=\tilde{f} \circ \tilde{g}$, for any $f: p^{*} V^{\prime} \otimes f^{*} \mathcal{E}^{\prime} \rightarrow$ $p^{*} V^{\prime \prime} \otimes f^{*} \mathcal{E}^{\prime \prime}$ and $g: p^{*} V \otimes f^{*} \mathcal{E} \rightarrow p^{*} V^{\prime} \otimes f^{*} \mathcal{E}^{\prime}$. Moreover, if $f=p^{*}\left(f_{1}\right) \otimes f_{2}$ for some $f_{1}: V \rightarrow V^{\prime}$ and $f_{2}: f^{*} \mathcal{E} \rightarrow f^{*} \mathcal{E}^{\prime}$, then $\tilde{f}=q^{*}\left(f_{1}\right) \otimes \tilde{f}_{2}$. Finally, if $V=V^{\prime}$ and $f=\operatorname{Id} \otimes f^{*}\left(f_{2}\right)$ for some $f_{2}: \mathcal{E} \rightarrow \mathcal{E}^{\prime}$, then $\tilde{f}=\operatorname{Id} \otimes f^{*} F\left(f_{2}\right)$. 
To conclude the proof of Theorem 2.8 , we have to prove that $F_{S}$ satisfies 1) and 2).

Proposition 2.10. One has natural isomorphisms:

a) $\tilde{F}\left(R_{\bullet}(M)\right) \otimes q^{*} N \simeq \tilde{F}\left(R_{\bullet}\left(M \otimes p^{*} N\right)\right)$ and

b) $f_{*} \tilde{F}\left(R_{\bullet}(M)\right) \simeq F\left(f_{*} R_{\bullet}(M)\right)$ (see Remark 2.3 for the definition of $F\left(f_{*} R_{\bullet}(M)\right)$ ).

Proof. a) Completely analogous arguments to that of Proposition 2.4 yield isomorphisms $\tilde{F}\left(R_{\mathcal{E}}\left(M \otimes p^{*} V\right) \simeq \tilde{F}\left(R_{\mathcal{E}}(M)\right) \otimes q^{*} V\right.$ and $\tilde{F}\left(R_{\mathcal{E}_{1}} R_{\mathcal{E}_{0}}\left(M \otimes p^{*} V\right)\right) \simeq \tilde{F}\left(R_{\mathcal{E}_{1}} R_{\mathcal{E}_{0}}(M)\right) \otimes$ $q^{*} V$. One checks that these isomorphisms are compatible with the differentials.

b) Completely analogous arguments to that of Proposition 2.5 yield isomorphisms $f_{*} \tilde{F}\left(R_{\mathcal{E}}(M)\right) \simeq F\left(f_{*} R_{\mathcal{E}}(M)\right)$ and $f_{*} \tilde{F}\left(R_{\mathcal{E}_{1}} R_{\mathcal{E}_{0}}(M)\right) \simeq F\left(f_{*} R_{\mathcal{E}_{1}} R_{\mathcal{E}_{0}}(M)\right)$. Again, one checks that these isomorphisms are compatible with the differentials.

It follows immediately that $F_{S}$ satisfies 1 ). For 2), one has

$$
\begin{array}{r}
f_{*} F_{S}(M)=f_{*} \operatorname{Coker}\left(\tilde{F}\left(R_{\bullet}(M)\right)\right)=\operatorname{Coker}\left(f_{*} \tilde{F}\left(R_{\bullet}(M)\right)\right) \stackrel{2.10}{\simeq} \operatorname{Coker} F\left(f_{*} R_{\bullet}(M)\right) \\
\stackrel{2.5}{\simeq} \operatorname{Coker} F\left(R_{\bullet}\left(f_{*} M\right)\right)
\end{array}
$$

Finally, Coker $F\left(R_{\bullet}\left(f_{*} M\right)\right) \simeq F\left(f_{*} M\right)$ by Proposition 2.2 and Remark 2.3.

This concludes the proof of Theorem 2.8.

Remark 2.11. The lifting $F_{S}$ of $F$ is functorial but it is not unique. Let us show an alternative lifting $F_{S}^{\prime}$. Instead of considering the "resolution" $R_{1}(M) \rightarrow R_{0}(M)$, let us consider the complex of objects in $D(X \times S)$

$$
R_{\bullet}(M):=\left\{\cdots \rightarrow R_{n}(M) \rightarrow R_{n-1}(M) \rightarrow \cdots \rightarrow R_{1}(M) \rightarrow R_{0}(M)\right\}
$$

where $R_{n}(M)=R_{0}\left(R_{n-1}(M)\right)$ and the differential $R_{n}(M) \rightarrow R_{n-1}(M)$ is the alternate sum of the $n+1$ natural maps from $R_{n}(M)$ to $R_{n-1}(M)$. As in the proof of the theorem, we can define $\tilde{F}\left(R_{\bullet}(M)\right)$, which is a complex of objects in $D_{\text {perf }}(Y \times S)^{*}$. Then one defines $F_{S}^{\prime}(M)$ as the "simple complex" associated to $\tilde{F}(R \cdot(M))$, i.e., for any $N \in D_{\text {perf }}(Y \times S)$ we define $F_{S}^{\prime}(M)(N)$ as the simple complex associated to the complex of vector spaces

$$
\cdots \rightarrow H\left(\tilde{F}\left(R_{n}(M)\right)(N)\right) \rightarrow H\left(\tilde{F}\left(R_{n}(M)\right)(N)\right) \rightarrow \cdots \rightarrow H\left(\tilde{F}\left(R_{0}(M)\right)(N)\right)
$$

This functor $F_{S}^{\prime}$ also satisfies properties 1), 2) and 3). Moreover, it has an extra "exact" property: first notice that $F_{S}^{\prime}(M)$ is in fact a functor from $D_{\text {perf }}(Y \times S)$ to the category of complexes of vector spaces (i.e, if $h: N \rightarrow N^{\prime}$ is a morphism in $D_{\text {perf }}(Y \times S)$, then $F_{S}^{\prime}(M)(h)$ is a morphism of complexes); the exact property is the following: if $N_{1} \rightarrow N_{2} \rightarrow N_{3}$ is an exact triangle in $D_{\text {perf }}(Y \times S)$, then

$$
F_{S}^{\prime}(M)\left(N_{1}\right) \rightarrow F_{S}^{\prime}(M)\left(N_{2}\right) \rightarrow F_{S}^{\prime}(M)\left(N_{3}\right)
$$

is an exact sequence of complexes (but may be not an exact triangle).

Let us see now how the extension theorem yields the integrality theorem.

Theorem 2.12. Let $X$ and $Y$ be two proper $k$-schemes and $F: D_{\text {perf }}(X) \rightarrow D_{\text {perf }}(Y)^{*}$ a k-linear graded functor. Then there exists an object $\omega$ in $D_{\text {perf }}(X \times Y)^{*}$ such that $F \simeq \Phi_{\omega}$ 
Proof. Let $F_{S}: D_{\text {fhd } / X, S}(X \times S) \rightarrow D_{\text {perf }}(Y \times S)^{*}$ be the $S$-linear functor given by Theorem 2.8. Take $S=X, f=p, \delta: X \rightarrow X \times S$ the diagonal map and $\mathcal{O}_{\Delta}=\delta_{*} \mathcal{O}_{X}$. Notice that $\mathcal{O}_{\Delta}$ is fhd over both $X$ and $S$. Then, by properties 1) and 2) of $F_{S}$,

$$
F(M) \simeq F\left(f_{*}\left(\mathcal{O}_{\Delta} \otimes p^{*} M\right)\right) \simeq f_{*} F_{S}\left(\mathcal{O}_{\Delta} \otimes p^{*} M\right) \simeq f_{*}\left(F_{S}\left(\mathcal{O}_{\Delta}\right) \otimes q^{*} M\right)
$$

So it is enough to take $\omega=F_{S}\left(\mathcal{O}_{\Delta}\right)$.

Since $F_{X}$ is functorial on $F$ we obtain:

Corollary 2.13. One has a functor

$$
\begin{aligned}
\Psi: \operatorname{Hom}_{k}\left(D_{\text {perf }}(X), D_{\text {perf }}(Y)^{*}\right) & \rightarrow D_{\text {perf }}(X \times Y)^{*} \\
F & \mapsto F_{X}\left(\mathcal{O}_{\Delta}\right)
\end{aligned}
$$

and the composition $\Phi \circ \Psi$ is isomorphic to the identity.

\subsection{Exactness.}

Definition 2.14. A linear form, $\omega: D_{\text {perf }}(X \times Y) \rightarrow D(k)$, on $X \times Y$ is said to be exact and perfect on $Y$ if for any $M \in D_{\text {perf }}(X)$, the functor $\Phi_{\omega}(M): D_{\text {perf }}(Y) \rightarrow D(k)$ is exact and perfect, i. e., $\Phi_{\omega}(M) \in D(Y)^{\vee}$.

We shall denote $D(X \times Y)^{Y-\vee}$ the full subcategory of $D(X \times Y)^{*}$ whose objects are the linear forms on $X \times Y$ which are exact and perfect on $Y$.

Taking into account that $D_{c}^{b}(Y) \rightarrow D_{\text {perf }}(Y)^{\vee}$ is an equivalence ( $Y$ projective), we obtain

Corollary 2.15. If $Y$ is projective, one has functors

$$
\begin{aligned}
\Phi: D_{\text {perf }}(X \times Y)^{Y-\vee} & \rightarrow \operatorname{Hom}_{k}\left(D_{\text {perf }}(X), D_{c}^{b}(Y)\right) \\
\omega & \mapsto \Phi_{\omega}
\end{aligned}
$$

and

$$
\begin{aligned}
\Psi: \operatorname{Hom}_{k}\left(D_{\text {perf }}(X), D_{c}^{b}(Y)\right) & \rightarrow D_{\text {perf }}(X \times Y)^{Y-\vee} \\
F & \mapsto F_{X}\left(\mathcal{O}_{\Delta}\right)
\end{aligned}
$$

and the composition $\Psi \circ \Phi$ is isomorphic to the identity.

A linear form $\omega$ on $X \times Y$ also defines an integral functor in the opposite direction (i.e., from $Y$ to $X$ ), which we shall denote by $\bar{\Phi}_{\omega}: D_{\text {perf }}(Y) \rightarrow D_{\text {perf }}(X)^{*}$.

Proposition 2.16. Assume that $X$ and $Y$ are projective. Let $\omega$ be a linear form on $X \times Y$. The following conditions are equivalent:

(1) $\omega$ is exact and perfect on $Y$ and the functor $\Phi_{\omega}: D_{\text {perf }}(X) \rightarrow D_{\text {perf }}(Y)^{\vee} \simeq D_{c}^{b}(Y)$ is exact.

(2) $\omega$ is exact and perfect on $X$ and the functor $\bar{\Phi}_{\omega}: D_{\text {perf }}(Y) \rightarrow D_{\text {perf }}(X)^{\vee} \simeq$ $D_{c}^{b}(X)$ is exact.

Proof. For any $M \in D_{\text {perf }}(X), N \in D_{\text {perf }}(Y)$, one has $\Phi_{\omega}(M)(N)=\bar{\Phi}_{\omega}(N)(M)$. Let us see that $(1) \Rightarrow(2)$.

From the equality $\Phi_{\omega}(M)(N)=\bar{\Phi}_{\omega}(N)(M)$ and (1) it follows immediately that $\omega$ is exact and perfect on $X$. It remains to prove that $\bar{\Phi}_{\omega}: D_{\text {perf }}(Y) \rightarrow D_{\text {perf }}(X)^{\vee} \simeq D_{c}^{b}(X)$ is exact.

For each object $\mathcal{E} \in D_{\text {perf }}(Y)$, let us denote $\mathcal{E}^{\#}:=\mathbf{R} \mathcal{H} o m_{Y}^{\bullet}\left(\mathcal{E}, q^{\prime} k\right)$. The functor $H: D_{\text {perf }}(X) \rightarrow \operatorname{Vect}(k)$ defined by $H(M)=\operatorname{Hom}_{D(Y)}\left(\Phi_{\omega}(M), \mathcal{E}^{\#}\right)$ is a contravariant 
cohomological functor of finite type, hence it is representable by an object $\Phi_{\omega}^{\#}(M) \in$ $D_{c}^{b}(X)$. Hence we obtain a pseudo right adjoint of $\Phi_{\omega}$ :

$$
\Phi_{\omega}^{\#}: D_{\text {perf }}^{\#}(Y) \rightarrow D_{c}^{b}(X)
$$

where $D_{\text {perf }}^{\#}(Y)$ is the full subcategory of $D_{c}^{b}(Y)$ whose objects are of the form $\mathcal{E}^{\#}$, with $\mathcal{E} \in D_{\text {perf }}(Y)$. That is, one has

$$
\operatorname{Hom}_{D(Y)}\left(\Phi_{\omega}(M), \mathcal{E}^{\#}\right)=\operatorname{Hom}_{D(X)}\left(M, \Phi_{\omega}^{\#}\left(\mathcal{E}^{\#}\right)\right)
$$

for any $M \in D_{\text {perf }}(X), \mathcal{E} \in D_{\text {perf }}(Y)$. Now, since $\Phi_{\omega}$ is exact, $\Phi_{\omega}^{\#}$ is also exact (one can copy the same proof than [1, Lemma 4.11]). Finally, it is easy to see that the equality $\Phi_{\omega}(M)(N)=\bar{\Phi}_{\omega}(N)(M)$ implies that $\bar{\Phi}_{\omega}(N)=\left[\Phi_{\omega}^{\#}\left(N^{\#}\right)\right]^{\#}$. Hence $\bar{\Phi}_{\omega}$ is exact.

Definition 2.17. A linear form $\omega: D_{\text {perf }}(X \times Y) \rightarrow D(k)$ is called bi-exact if it satisfies any of the equivalent conditions of Proposition 2.16.

We denote by $D_{\text {perf }}(X \times Y)^{\text {bi- } \vee}$ the full subcategory $D_{\text {perf }}(X \times Y)^{*}$ whose objects are the bi-exact linear forms on $X \times Y$. We have then embeddings

$D_{c}^{b}(X \times Y) \simeq D_{\text {perf }}(X \times Y)^{\vee} \hookrightarrow D_{\text {perf }}(X \times Y)^{\mathrm{bi}-\vee} \hookrightarrow D_{\text {perf }}(X \times Y)^{Y-\vee} \hookrightarrow D_{\text {perf }}(X \times Y)^{*}$

Finally, for bi-exact linear forms we have:

Corollary 2.18. Assume that $X$ and $Y$ are projective. One has functors

$$
\begin{aligned}
\Phi: D_{\text {perf }}(X \times Y)^{\mathrm{bi}-\vee} & \rightarrow \operatorname{Hom}_{k}^{\text {ex }}\left(D_{\text {perf }}(X), D_{c}^{b}(Y)\right) \\
\omega & \mapsto \Phi_{\omega}
\end{aligned}
$$

and

$$
\begin{aligned}
\Psi: \mathbf{H o m}_{k}^{\text {ex }}\left(D_{\text {perf }}(X), D_{c}^{b}(Y)\right) & \rightarrow D_{\text {perf }}(X \times Y)^{b i-\vee} \\
F & \mapsto F_{X}\left(\mathcal{O}_{\Delta}\right)
\end{aligned}
$$

and the composition $\Psi \circ \Phi$ is isomorphic to the identity.

\section{Relative Integral Functors}

In this section we shall reproduce the main results of the previous section for relative schemes. Let $p: X \rightarrow T$ and $q: Y \rightarrow T$ be two proper $T$-schemes. Let us still denote by $p: X \times_{T} Y \rightarrow Y$ and $q: X \times_{T} Y \rightarrow Y$ the natural morphisms. For each object $K \in D_{c}^{b}\left(X \times_{T} Y\right)$ one has the (relative) exact integral functor

$$
\begin{aligned}
\Phi_{K}: D_{\text {perf }}(X) & \rightarrow D_{c}^{b}(Y) \\
M & \mapsto p_{*}\left(K \otimes q^{*} M\right)
\end{aligned}
$$

This functor is $T$-linear: for any $M \in D_{\text {perf }}(X), \mathcal{E} \in D_{\text {perf }}(T)$ one has a natural isomorphism $\Phi_{K}\left(M \otimes p^{*} \mathcal{E}\right) \simeq \Phi_{K}(M) \otimes q^{*} \mathcal{E}$.

If we replace $K$ by an object $\omega \in D_{\text {perf }}\left(X \times_{T} Y\right)^{*}$, then we have a functor

$$
\begin{aligned}
\Phi_{\omega}: D_{\text {perf }}(X) & \rightarrow D_{\text {perf }}(Y)^{*} \\
M & \mapsto p_{*}\left(\omega \otimes q^{*} M\right)
\end{aligned}
$$

which is also $T$-linear (in a natural sense, see below). This will be called a (relative non-exact) integral functor. Our aim is to show that (under flatness hypothesis of $p$ and $q$ ) any $T$-linear functor $D_{\text {perf }}(X) \rightarrow D_{\text {perf }}(Y)^{*}$ is integral, i.e., it is isomorphic to $\Phi_{\omega}$ for some $\omega \in D_{\text {perf }}\left(X \times_{T} Y\right)^{*}$.

We shall first give some natural definitions about $T$-linear categories and functors. 
Definition 3.1. Let $T$ be a scheme. A $T$-linear structure on an additive graded category $\mathcal{D}$ is a biadditive and bigraded functor

$$
\begin{aligned}
D_{\text {perf }}(T) \times \mathcal{D} & \rightarrow \mathcal{D} \\
(\mathcal{E}, P) & \mapsto \mathcal{E} \otimes P
\end{aligned}
$$

satisfying functorial isomorphisms:

(1) $\phi_{P}: \mathcal{O}_{T} \otimes P \simeq P$.

(2) $\psi_{\mathcal{E}_{1}, \mathcal{E}_{2}, P}: \mathcal{E}_{1} \otimes\left(\mathcal{E}_{2} \otimes P\right) \simeq\left(\mathcal{E}_{1} \stackrel{\mathrm{L}}{\otimes} \mathcal{O}_{T} \mathcal{E}_{2}\right) \otimes P$.

Definition 3.2. A $T$-linear category is a graded category endowed with a $T$-linear structure. A $T$-linear functor $F: \mathcal{D} \rightarrow \mathcal{D}^{\prime}$ between $T$-linear categories is a functor endowed with a bi-additive and bi-graded bi-functorial isomorphism $\theta_{F}(P, E): F(\mathcal{E} \otimes$ $P) \simeq \mathcal{E} \otimes F(P), \mathcal{E} \in D_{\text {perf }}(T), P \in \mathcal{D}$, which is compatible with $\phi_{P}$ and $\psi_{\mathcal{E}_{1}, \mathcal{E}_{2}, P}$ in the obvious sense. That is, a $T$-linear functor is a pair $\left(F, \theta_{F}\right)$, though we shall usually denote it by $F$.

A $T$-linear morphism $\phi: F \rightarrow F^{\prime}$ between $T$-linear functors is a morphism of functors which is compatible with the $\theta^{\prime} s$, i.e., such that the diagram

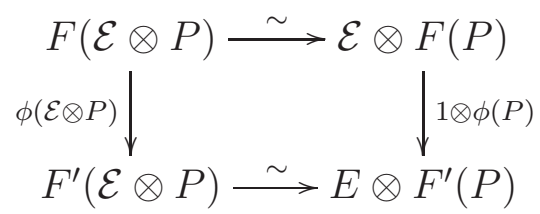

is commutative.

Is $S=$ Spec $k$, the above notion of $k$-linear category coincides with the usual notion of $k$-linear graded category.

We shall denote by $\operatorname{Hom}_{T}\left(\mathcal{D}, \mathcal{D}^{\prime}\right)$ the category of $T$-linear functors from $\mathcal{D}$ to $\mathcal{D}^{\prime}$ and $T$-linear morphisms. It has a natural $T$-linear structure. If $\mathcal{D}$ and $\mathcal{D}^{\prime}$ are triangulated categories, we shall denote by $\operatorname{Hom}_{T}^{\text {ex }}\left(\mathcal{D}, \mathcal{D}^{\prime}\right)$ the full subcategory of exact $T$-linear functors.

Example 3.3. If $p: X \rightarrow T$ is a $T$-scheme, then $D_{\text {perf }}(X)$ (or $D(X), D_{c}^{b}(X)$ ) has a natural $T$-linear structure, namely: $\mathcal{E} \otimes P:=p^{*} \mathcal{E} \otimes_{\mathcal{O}_{X}} P$. If $q: Y \rightarrow T$ is another $T$-scheme and $K \in D_{c}^{b}\left(X \times_{T} Y\right)$, then the integral functor $\Phi_{K}: D_{\text {perf }}(X) \rightarrow D_{\text {perf }}(Y)$ is a $T$-linear functor (with the $\theta$ induced by the projection formula).

For any $k$-linear category $\mathcal{D}$, we shall denote $\mathcal{D}^{*}=\operatorname{Hom}_{k}(\mathcal{D}, D(k))$. If $\mathcal{D}$ is a triangulated category, we shall denote $\mathcal{D}^{\vee}=\operatorname{Hom}_{k}^{\text {ex }}\left(\mathcal{D}, D_{\text {perf }}(k)\right)$. If $\mathcal{D}$ is a $T$-linear category, then $\mathcal{D}^{*}$ has a natural $T$-linear structure, defining $(\mathcal{E} \otimes \omega)(M)=\omega(\mathcal{E} \otimes M)$. Moreover, if $\mathcal{D}$ is triangulated and $\mathcal{E} \otimes(-): \mathcal{D} \rightarrow \mathcal{D}$ is exact for any $\mathcal{E} \in D_{\text {perf }}(T)$, then $D^{\vee}$ is also a $T$-linear category.

For any $T$-scheme $p: Z \rightarrow T$, the equivalence $D_{c}^{b}(Z) \stackrel{\sim}{\rightarrow} D_{\operatorname{perf}}(Z)^{*}$ of Proposition 1.2 is $T$-linear ( $Z$ is a projective $k$-scheme).

Let $X$ and $Y$ be two $T$-schemes and $\omega: D_{\text {perf }}\left(X \times_{T} Y\right) \rightarrow D(k)$ a $k$-linear form on $X \times_{T} Y$. Let us denote $p: X \times_{T} Y \rightarrow X$ and $q: X \times_{T} Y \rightarrow Y$ the natural projections. For each $M \in D_{\text {perf }}(X)$ we have a $k$-linear functor

$$
\Phi_{\omega}(M): D_{\text {perf }}(Y) \rightarrow D(k)
$$


defined by $\Phi_{\omega}(M)(N)=\omega\left(p^{*} M \otimes q^{*} N\right)$. We have then a $T$-linear functor

$$
\Phi_{\omega}: D_{\text {perf }}(X) \rightarrow D_{\text {perf }}(Y)^{*}
$$

Definition 3.4. We say that $\Phi_{\omega}: D_{\text {perf }}(X) \rightarrow D_{\text {perf }}(Y)^{*}$ is a relative integral functor of kernel $\omega$.

Example 3.5. If $\omega$ is exact and perfect, then $\omega \simeq \omega_{K}$ for a unique $K \in D_{c}^{b}\left(X \times_{T} Y\right)$, $\Phi_{\omega}$ takes values in $D(Y)^{\vee} \simeq D_{c}^{b}(Y)$ and $\Phi_{\omega} \simeq \Phi_{K}$.

The extension theorem has now the following form:

Theorem 3.6. Let $p: X \rightarrow T$ and $q: Y \rightarrow T$ be two proper and flat $T$-schemes. Let $F: D_{\text {perf }}(X) \rightarrow D_{\text {perf }}(Y)^{*}$ be a T-linear functor. For any proper and flat $T$-scheme $f: S \rightarrow T$ there exists a functor

$$
F_{S}: D_{\text {fhd } / X, S}\left(X \times_{T} S\right) \rightarrow D_{\text {perf }}\left(Y \times_{T} S\right)^{*}
$$

such that:

1) $F_{S}$ is $S$-linear: one has a bi-functorial isomorphism $F_{S}\left(M \otimes p^{*} N\right) \simeq F_{S}(M) \otimes q^{*} N$, for any $M \in D_{\text {fhd } / X, S}\left(X \times_{T} S\right), N \in D_{\text {perf }}(S)$.

2) It is compatible with $F$ : for any $M \in D_{\text {fhd } / X, S}\left(X \times_{T} S\right)$ one has a natural isomorphism $f_{*} F_{S}(M) \simeq F\left(f_{*} M\right)$.

3) $F_{S}$ is functorial on $F$.

Proof. The proof is completely analogous to that of Theorem 2.8. One constructs the relative version of the "perfect resolution" of section 2.2 , just replacing $k$ by $T$. The $T$ linearity of $F$ is necessary to reproduce Lemma 2.9 in the relative setting. The flatness hypothesis is necessary for the use of flat base change and 1) of Proposition 2.7.

As in the absolute case, we obtain corollaries:

Corollary 3.7. One has a T-linear functor

$$
\begin{aligned}
\Psi: \operatorname{Hom}_{T}\left(D_{\text {perf }}(X), D_{\text {perf }}(Y)^{*}\right) & \rightarrow D_{\text {perf }}\left(X \times_{T} Y\right)^{*} \\
F & \mapsto F_{X}\left(\mathcal{O}_{\Delta}\right)
\end{aligned}
$$

and the composition $\Phi \circ \Psi$ is isomorphic to the identity.

Corollary 3.8. Assume that $X, Y$ and $T$ are projective $k$-schemes. One has $T$-linear functors

$$
\begin{aligned}
\Phi: D_{\text {perf }}\left(X \times_{T} Y\right)^{\mathrm{bi}-\vee} & \rightarrow \mathbf{H o m}_{T}^{\mathrm{ex}}\left(D_{\text {perf }}(X), D_{c}^{b}(Y)\right) \\
\omega & \mapsto \Phi_{\omega}
\end{aligned}
$$

and

$$
\begin{aligned}
\Psi: \operatorname{Hom}_{T}^{\text {ex }}\left(D_{\text {perf }}(X), D_{c}^{b}(Y)\right) & \rightarrow D_{\text {perf }}\left(X \times_{T} Y\right)^{\mathrm{bi}-\vee} \\
F & \mapsto F_{X}\left(\mathcal{O}_{\Delta}\right)
\end{aligned}
$$

and the composition $\Psi \circ \Phi$ is isomorphic to the identity.

\section{ReFEREnCES}

[1] M.R. BAllard, Derived categories of sheaves on quasi-projective schemes, arXiv:0801.2599v2.

[2] A. Bondal. M. Larsen, V. Lunts, Grothendieck ring of pretriangulated categories, Int. Math. Res. Not., 29 (2004), pp 1461-1495.

[3] A. Bondal And M. VAn DEN BERGH, Generators and representability of functors in commutative and noncommutative geometry, Mosc. Math. J., 3 (2003), pp. 1-36. 
[4] A. Caldararu, Derived categories of sheaves: askimming, Snowbird lectures in algebraic geometry, 43-75, Contemp. Math. 388, Amer. Math. Soc. Providence RI (2005).

[5] A. Canonaco and P. Stellari, Twisted fourier-mukai functors, Adv. Math., 212 (2007), pp. $484-503$.

[6] A. Canonaco and P. Stellari, Fourier-Mukai functors in the supported case, arXiv 1010.0798.

[7] A. Canonaco and P. Stellari, Non-uniqueness of Fourier-Mukai kernels, arXiv 1009.5577.

[8] D. Hernández Ruipérez, A.C. López Martín and F. Sancho de Salas, Fourier-Mukai transforms for Gorenstein Schemes, Adv. in Maths., 211 (2007), pp. 594-620.

[9] V. Lunts, D. O. ORLov, Uniqueness of enhancements for triangulated categories, J. Amer. Math. Soc, 23 (2010), pp. 853-908.

[10] Y. KaWamata, Equivalences of derived categories of sheaves on smooth stacks, Amer. J. Math., 126 (2004), pp. 1057-1083.

[11] D. O. OrLov, Equivalences of derived categories and K3 surfaces, J. Math. Sci. (New York), 84 (1997), pp. 1361-1381. Algebraic geometry, 7.

[12] C. Sancho De Salas And F. Sancho DE Salas, The linear dual of the derived category of a scheme, Proc. Amer. Math. Soc. (to appear).

[13] B. TöEn, The homology theory of dg-categories and derived Morita theory, Invent. Math, 167 (2007), pp. 615-667.

Departamento de Matemáticas e Instituto Universitario de Física Fundamental y Matemáticas (IUfFym), Universidad de Salamanca, Plaza de la Merced 1-4, 37008 Salamanca, Spain

E-mail address: fsancho@usal.es 